Research Article

\title{
PERK-Dependent Activation of the JAK2/STAT3 Pathway Contributes to High Glucose-Induced Extracellular Matrix Deposition in Renal Tubular Epithelial Cells
}

\author{
Yan Bao $\mathbb{D},{ }^{1}$ Wei Liang $\mathbb{D},{ }^{2}$ Yingchun Ye $\mathbb{D},{ }^{1}$ and Bo Yi $\mathbb{D}^{1}$ \\ ${ }^{1}$ Department of Endocrinology, Renmin Hospital of Wuhan University, Wuhan 430060, Hubei, China \\ ${ }^{2}$ Department of Nephrology, Renmin Hospital of Wuhan University, Wuhan 430060, Hubei, China \\ Correspondence should be addressed to Bo Yi; dumei1223@126.com
}

Received 8 May 2021; Accepted 5 July 2021; Published 20 July 2021

Academic Editor: Guglielmo Stabile

Copyright (C) 2021 Yan Bao et al. This is an open access article distributed under the Creative Commons Attribution License, which permits unrestricted use, distribution, and reproduction in any medium, provided the original work is properly cited.

\begin{abstract}
Background. Although the deposition of extracellular matrix (ECM) is critical leading to tubular damage in diabetic kidney disease (DKD), the mechanism still remains unclear. The purpose of this study was to demonstrate a role for protein kinase R-like endoplasmic reticulum kinase (PERK) (a protein located in the endoplasmic reticulum membrane) in this pathologic process. Methods. NRK-52E cells were grown in the media containing different concentrations of glucose or thapsigargin for different durations. Cells were subsequently incubated with or without AG490, a selective inhibitor of Janus kinase 2 (JAK2) or GSK2606414 (a selective PERK inhibitor). We evaluated the production of TGF- $\beta 1$, fibronectin, and collagen I proteins by ELISA. The levels of $78 \mathrm{kD}$-glucose-regulated protein (GRP78) and PERK, as well as the phosphorylation statues of PERK and JAK2/ signal transducer and activator of transcription (STAT3), were determined by western blotting analysis. Results. We showed that the increased phosphorylation of JAK2 and STAT3 was accompanied by overexpression of TGF- $\beta 1$ and ECM deposition in high glucose medium. Disruption of the JAK2/STAT3 pathway with AG490 significantly prevents the high glucose-induced increase in TGF- $\beta 1$, fibronectin, and collagen I. High glucose induced the overproduction of GRP78 and phosphorylation of PERK, which indicated that endoplasmic reticulum stress (ERS) was triggered in NRK-52E cells cultured under high glucose condition. Inhibition of PERK phosphorylation with GSK2606414, however, blocked the effect of JAK2/STAT3 on the production of TGF- $\beta 1$ and ECM components in NRK-52E cells. Conclusion. Our data indicated that the ECM accumulation induced by high glucose arouse via the PERK-dependent JAK2/STAT3-signaling pathway in renal tubular epithelial cells.
\end{abstract}

\section{Introduction}

Diabetic kidney disease (DKD) is a serious chronic microvascular complication of diabetes and is the leading cause of end-stage renal disease (ESRD) [1]. Although DKD is characterized by glomerular and tubular epithelial hypertrophy and the accumulation of extracellular matrix (ECM), the severity of tubulointerstitial lesions is more closely related to proteinuria and a progressive diminution in renal function. Some diabetic patients still develop progressive renal damage even though their metabolic abnormalities are well controlled. One of the mechanisms underlying such damage is hypothesized to be the initiation of tubular epithelial cell injury by high glucose concentration, accompanied by the overproduction of TGF- $\beta 1$ and ECM components, such as collagen and fibronectin (FN) [2]. Therefore, preventing the accumulation of ECM in the tubulointerstitium is critical to improving the accelerated progression of DKD.

The most potent simulator of ECM accumulation is transforming growth factor- $\beta 1$ (TGF- $\beta 1$ ), a cardinal profibrotic member of the TGF- $\beta$ superfamily [3]. This is considered to be an essential factor in the development of DKD in diabetic animals and patients [4]. Wang et al. [5] have revealed that high glucose can stimulate TGF- $\beta 1$ in rat glomerular mesangial cells through the Janus kinase 2 (JAK)/signal transducer and activator of transcription (STAT) pathway. Experimental evidence suggests that 
inhibition of JAK/STAT (in particular, JAK2/STAT3) may suppress renal fibrosis and thereby protect renal function [6]. The JAK/STAT signaling pathway, activated by its phosphorylation, is closely related to the production of TGF$\beta 1, \mathrm{FN}$, and collagen [7]. Fibronectin and collagen are the primary components of the ECM. The FN protein is composed of two peptide chains that attach cells to a variety of ECM components [8]. However, research on the role of JAK/STAT signaling in renal damage has been inconsistent.

Endoplasmic reticulum stress (ERS) has been widely indicated to play an essential role in kidney injury $[9,10]$. The endoplasmic reticulum (ER) is the processing center where newly synthesized proteins are correctly folded and assembled. Adverse stimuli such as hyperglycemia, oxidative stress, and hypoxia can lead to the accumulation of unfolded or misfolded proteins in the ER lumen and ultimately drive ER stress, which is recognized to be an adaptive response [11]. However, when ERS is prolonged and severe, it can lead to a variety of diseases, including DKD and acute kidney injury [12]. Malfunctions of the ER induced by a variety of factors can lead to the unfolded protein response (UPR), resulting in ERS. The UPR is initiated by three transmembrane transducers, one of which is protein kinase R-like endoplasmic reticulum kinase (PERK). The interactions between ERS and STAT signaling are currently receiving increasing attention. Recent evidence suggests that the PERK/JAK1/STAT3 signaling pathway is a driver of neuroinflammation and that this may be relevant to multiple sclerosis [13]. However, it remains unclear as to whether ERS can activate the JAK/STAT pathway in the kidney. In this study, we investigated the relationship between ERS and JAK/ STAT and explored their roles in high glucose-induced ECM deposition in renal tubular epithelial cells.

\section{Materials and Methods}

2.1. Experimental Materials. Anti-GRP78, PERK, phosphorPERK, JAK2, and STAT3 antibodies were purchased from Santa Cruz Biotechnology (Santa Cruz, CA, USA). We purchased thapsigargin from Sigma (USA) and GSK2606414 and AG490 from Selleck Chemicals (USA). TGF- $\beta 1$ and FN ELISA kits were bought from Boster (Wuhan, China), a collagen I ELISA kit was from BlueGene Biotech. (Shanghai, China), and a cell counting kit (CCK-8) was bought from Dojindo (Kumamoto, Japan). Rat renal proximal tubule epithelial cells (NRK-52E) were obtained from the American Type Culture Collection.

2.2. Cell Culture. NRK-52E cells were cultured in Dulbecco Modified Eagle's Medium (DMEM) supplemented with 10\% of fetal bovine serum, glucose $(5.6 \mathrm{mM}), 100 \mathrm{mg} / \mathrm{mL}$ of streptomycin, and 100 units $/ \mathrm{ml}$ of penicillin at $37^{\circ} \mathrm{C}$, in a $5 \%$ $\mathrm{CO}_{2}$ atmosphere. Cells were seeded at a density of $1 \times 10^{5}$ cells/well in 6-well dishes and were transferred to the serumfree medium for 24 hours for further experiments.

2.3. Enzyme-Linked Immunosorbent Assay. TGF- $\beta 1$, FN, and collagen I were examined using ELISA kits according to the manufacturers' instructions. Each experiment was repeated three times.
2.4. Western Blot Analysis. These methods were performed according to our previously published study [14]. After completing experiments, the medium was removed and the cells were washed twice with ice-cold PBS. Cells were then lysed with RIPA lysis buffer (Beyotime biotechnology, China), containing $1 \mathrm{mmol} / \mathrm{L}$ PMSF and quantified using a bicinchoninic acid protein assay kit (Beyotime Biotechnology, China). Cell homogenates were separated by $10 \%$ SDS-PAGE and transferred to polyvinylidene fluoride membranes, which were then blocked for $1 \mathrm{~h}$ at room temperature with 5\% skim milk powder in TBST. The blots were incubated with one of the following primary polyclonal antibodies: GRP78, phosphor-PERK, phosphor-JAK2, or phosphor-STAT3. Horseradish peroxidase-conjugated goat anti-rabbit immunoglobulin $G$ was used as the secondary antibody. After the chemiluminescence reaction, the bands were detected by exposing the blots to the X-ray film. The same membrane was then reused to determine $\beta$-actin by incubating it with a monoclonal anti- $\beta$-actin antibody. For quantitative analysis, the bands were detected and evaluated densitometrically with BandScan software (Glyko Inc., Novato, CA, USA) and normalized to the corresponding density of $\beta$-actin. Every result was issued from three independent experiments.

2.5. CCK-8 Assay. The cellular suspensions $(100 \mu \mathrm{L})$ were added to each well of a 96-well plate. After they adhered, cells were synchronized for $24 \mathrm{~h}$ and cultured with different concentrations of glucose. The medium was discarded, and the plate was washed twice with phosphate-buffered saline (PBS). Ten microliters of CCK-8 and $90 \mu \mathrm{L}$ fresh medium were added to each well of the plate. The cells were subsequently incubated for $4 \mathrm{~h}$ at $37^{\circ} \mathrm{C}$, and absorbance was measured at $450 \mathrm{~nm}$. Three independent experiments were performed.

2.6. Statistical Analysis. We expressed all data as means \pm SE. Data were compared among groups using one-way ANOVA followed by Tukey's multiple-comparison test with SPSS statistical software version 21.0 (SPSS Inc., Chicago, USA). A value of $P<0.05$ was considered statistically significant.

\section{Results}

3.1. Overexpression of TGF- $\beta 1, F N$, and Collagen I Induced by High Glucose Is JAK2/STAT3-Dependent in NRK-52E Cells. In this experiment, we cultured NRK-52E cells in different concentrations of glucose (NC group, $5.6 \mathrm{mM}$; H15 group, $15 \mathrm{mM}$; H25 group, $25 \mathrm{mM}$; and H50 group, $50 \mathrm{mM}$ ) or mannitol (Mann group, $5.6 \mathrm{mM} \mathrm{D}$-glucose $+19.4 \mathrm{mM}$ $\mathrm{D}$-mannitol) for $12 \mathrm{~h}, 24 \mathrm{~h}$, or $48 \mathrm{~h}$. The presence of higher levels of glucose augmented the levels of TGF- $\beta 1$ and the components of the ECM, while the expression of the above proteins did not change in the control or mannitol groups (Table 1). Because the concentrations of the aforementioned proteins were highest when cells were cultured in $25 \mathrm{mM}$ glucose for 24 hours, the subsequent experiments were selected on this basis (Table 1). 
TABLE 1: The protein levels of TGF- $\beta_{1}$, FN, and collagen I in different concentrations of glucose at different times.

\begin{tabular}{|c|c|c|c|c|c|c|}
\hline & \multicolumn{2}{|c|}{ TGF- $\beta 1$ (ng/L) } & \multicolumn{2}{|c|}{$\mathrm{FN}(\mu \mathrm{g} / \mathrm{ml})$} & \multicolumn{2}{|c|}{ Coll I (ng/L) } \\
\hline & Mean \pm SD & $P$ & Mean \pm SD & $P$ & Mean \pm SD & $P$ \\
\hline $\mathrm{NC} / 12 \mathrm{~h}$ & $1277.52 \pm 126.83$ & - & $578.46 \pm 62.22$ & - & $82.47 \pm 6.83$ & - \\
\hline Mann/12 h & $1259.70 \pm 154.35$ & 1.00 & $575.66 \pm 113.15$ & 1.00 & $85.50 \pm 8.81$ & 1.00 \\
\hline $\mathrm{H} 15 / 12 \mathrm{~h}$ & $1435.74 \pm 144.83$ & 0.99 & $1089.94 \pm 171.20^{*}$ & $<0.05$ & $123.99 \pm 15.18^{*}$ & $<0.05$ \\
\hline $\mathrm{H} 25 / 12 \mathrm{~h}$ & $1912.33 \pm 244.32^{*}$ & 0.02 & $1185.45 \pm 252.14^{*}$ & $<0.05$ & $163.42 \pm 10.55^{*}$ & $<0.05$ \\
\hline $\mathrm{H} 50 / 12 \mathrm{~h}$ & $1591.20 \pm 200.31$ & 0.76 & $1042.22 \pm 119.98^{*}$ & 0.03 & $149.20 \pm 8.90^{*}$ & $<0.05$ \\
\hline $\mathrm{NC} / 24 \mathrm{~h}$ & $1280.71 \pm 174.95$ & - & $659.93 \pm 76.47$ & - & $87.54 \pm 3.37$ & - \\
\hline Mann/24h & $1267.80 \pm 141.28$ & 1.00 & $593.29 \pm 100.33$ & 1.00 & $94.36 \pm 12.63$ & 1.00 \\
\hline $\mathrm{H} 15 / 24 \mathrm{~h}$ & $1899.81 \pm 192.74^{\dagger}$ & 0.02 & $1242.78 \pm 157.80^{\dagger}$ & $<0.05$ & $137.62 \pm 8.23^{\dagger}$ & $<0.05$ \\
\hline $\mathrm{H} 25 / 24 \mathrm{~h}$ & $2002.32 \pm 227.84^{\dagger}$ & $<0.05$ & $1499.52 \pm 178.26^{\dagger}$ & $<0.05$ & $205.16 \pm 9.35^{\dagger}$ & $<0.05$ \\
\hline $\mathrm{H} 50 / 24 \mathrm{~h}$ & $1461.57 \pm 204.17$ & 0.99 & $1281.24 \pm 185.36^{\dagger}$ & $<0.05$ & $167.17 \pm 14.56^{\dagger}$ & $<0.05$ \\
\hline $\mathrm{NC} / 48 \mathrm{~h}$ & $1190.83 \pm 88.05$ & - & $604.00 \pm 93.41$ & - & $89.97 \pm 9.13$ & - \\
\hline Mann/48 h & $1207.14 \pm 244.60$ & 1.00 & $590.15 \pm 75.43$ & 1.00 & $81.73 \pm 9.56$ & 1.00 \\
\hline $\mathrm{H} 15 / 48 \mathrm{~h}$ & $1351.34 \pm 208.61$ & 0.99 & $1145.16 \pm 164.25^{\ddagger}$ & $<0.05$ & $140.06 \pm 13.63^{\ddagger}$ & $<0.05$ \\
\hline $\mathrm{H} 25 / 48 \mathrm{~h}$ & $1813.77 \pm 216.90^{\ddagger}$ & 0.02 & $1286.16 \pm 180.00^{\ddagger}$ & $<0.05$ & $186.33 \pm 14.73^{\ddagger}$ & $<0.05$ \\
\hline $\mathrm{H} 50 / 48 \mathrm{~h}$ & $1301.38 \pm 176.79$ & 1.00 & $1047.77 \pm 52.37^{\ddagger}$ & 0.04 & $159.40 \pm 14.21^{\ddagger}$ & $<0.05$ \\
\hline
\end{tabular}

$N=3,{ }^{*} P<0.05$ compared with the NC group at $12 \mathrm{~h} ;{ }^{\dagger} P<0.05$ compared with the NC group at $24 \mathrm{~h},{ }^{\ddagger} P<0.05$ compared with the NC group at $48 \mathrm{~h}$.

Our results also showed that high glucose facilitated the phosphorylation of JAK2 and STAT3 $(P<0.05$, Figures $1($ a) and $1(\mathrm{~b}))$. To determine whether the overexpression of TGF$\beta 1$ and ECM induced by high glucose occurred by virtue of the JAK2/STAT3 pathway, we cocultured cells with AG490 (a selective inhibitor of JAK2) and observed that AG490 $(100 \mu \mathrm{M})$ suppressed high glucose-induced phosphorylation of JAK2/STAT3 $(P<0.05)$ (Figures $1(\mathrm{c})$ and $1(\mathrm{~d}))$ and reduced TGF- $\beta 1, \mathrm{FN}$, and collagen I protein levels $(P<0.05$, Figure 1(e)). This indicated that overproduction of TGF- $\beta 1$, FN, and collagen I induced by high glucose was JAK2/ STAT3-dependent in NRK-52E cells.

3.2. High Glucose Triggers ER Stress. Compared with the control group, although the expression of GRP78 protein and phosphorylation of PERK were enhanced under high glucose conditions $(P<0.05$, Figure 2$)$, PERK protein did not change. These results are congruent with our previous work, suggesting that ERS was triggered by high glucose [14].

3.3. Overexpression of TGF- $\beta 1$ and ECM Deposits during ERS Are JAK2/STAT3-Dependent. To investigate the role of the JAK2/STAT3 signaling axis on ERS-induced overproduction of TGF- $\beta 1$ and the components of the ECM, NRK-52E cells were treated with thapsigargin $(0.1 \mu \mathrm{M}$ and $0.2 \mu \mathrm{M})$ for $6 \mathrm{~h}, 24 \mathrm{~h}$, or $48 \mathrm{~h}$. Thapsigargin augmented protein levels for TGF- $\beta 1, \mathrm{FN}$, and collagen I (Table 2); when cells were incubated in $0.1 \mu \mathrm{M}$ thapsigargin for $24 \mathrm{~h}$, the levels of TGF- $\beta 1$ and ECM were higher than in the other groups (Table 2). In addition, thapsigargin accelerated the phosphorylation of JAK2 and STAT3 $(P<0.05$, Figures 3(a) and 3(b)). We also observed that, for cells cocultured with AG490 $(100 \mu \mathrm{M})$ and thapsigargin, AG490 inhibited the phosphorylation of JAK2 and STAT3 induced by thapsigargin $(P<0.05$, Figures $3(c)$ and $3(\mathrm{~d}))$, as well as the levels of TGF- $\beta 1$ protein and ECM $(P<0.05$, Figure 3(e)). Collectively, these results indicated that the overproduction of TGF- $\beta 1$ and ECM stimulated during ERS was mediated by the JAK2/STAT3 pathway.

3.4. The Activation of JAK2/STAT3 during ERS Is Partially PERK-Dependent. To determine the role of PERK in the activation of JAK2/STAT3, we pretreated cells with GSK2606414 (a potent selective inhibitor of PERK) and cocultured them with thapsigargin. GSK2606414 (100 nM) arrested the phosphorylation of JAK2 and STAT3 significantly $(P<0.05$, Figure 4$)$ accompanied by diminution in TGF- $\beta 1$ protein and ECM components $(P<0.05$, Figure 3(e)). These data suggested that the PERK branch of the UPR in ERS was important in facilitating the phosphorylation of JAK2 and STAT3.

3.5. High Glucose Upregulates the Deposit of ECM via PERKDependent JAK2/STAT3 in NRK-52E Cells. NRK-52E cells were pretreated with GSK2606414 (10 nM and $100 \mathrm{nM})$ for 30 minutes and coincubated with $25 \mathrm{mM}$ glucose. When cells were treated with GSK2606414 under a high glucose condition, the phosphorylation of JAK2/STAT3 was partially blocked $(P<0.05$, Figures 5(a) and 5(b)) along with TGF- $\beta 1, \mathrm{FN}$, and collagen I proteins $(P<0.05$, Figure $5(\mathrm{c}))$. Collectively, these results indicated that TGF- $\beta 1$ and ECM accumulation induced by high glucose was partially mediated via the PERK/JAK2/STAT3 pathway.

3.6. Cell Viability under Different Concentrations of Glucose. The NRK-52E cells were cultured in the medium with different concentrations of glucose or mannitol for $12 \mathrm{~h}, 24 \mathrm{~h}$, or $48 \mathrm{~h}$. Compared with the control group, the results of the CCK8 assay indicated an attenuation of cellular activity in the $\mathrm{H} 25$ group at $48 \mathrm{~h}$ and in the $\mathrm{H} 50$ group at $24 \mathrm{~h}$ and $48 \mathrm{~h}(P<0.05$, Figure 6(a)). We did not observe a reduction in cellular viability in the $\mathrm{H} 25$ group at $12 \mathrm{~h}$ and $24 \mathrm{~h}$ or in the $\mathrm{H} 15$ group for different hours. Then, NRK-52E cells were pretreated with GSK2606414 (10 nM and $100 \mathrm{nM})$ for 30 minutes and 

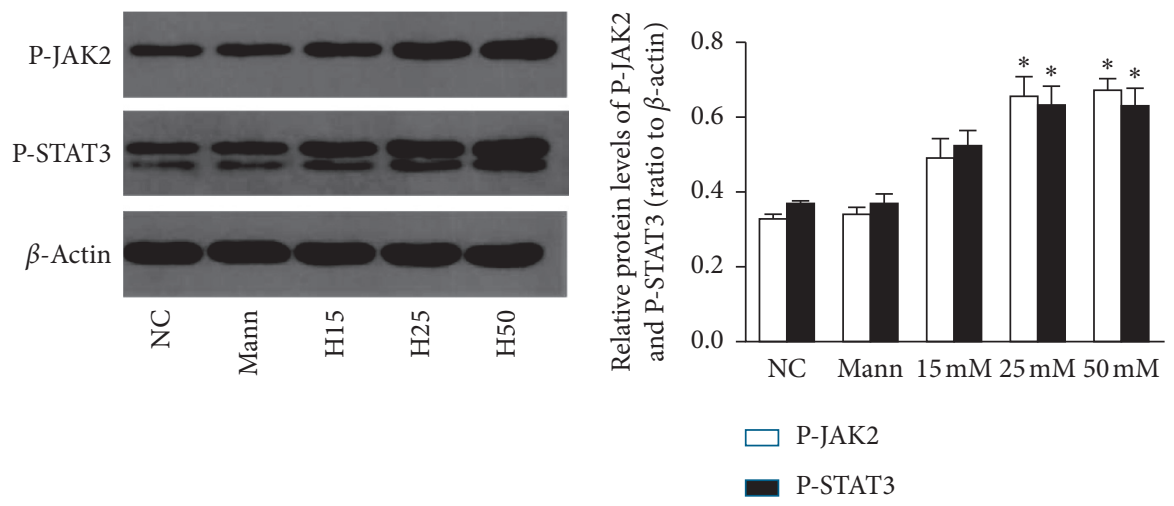

(a)

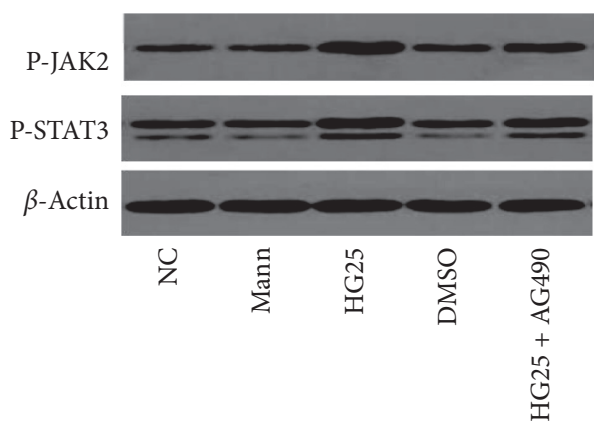

(b)

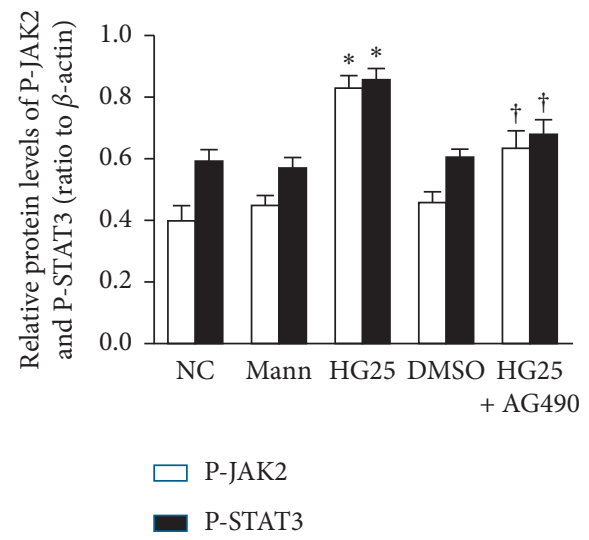

(d)

(c)

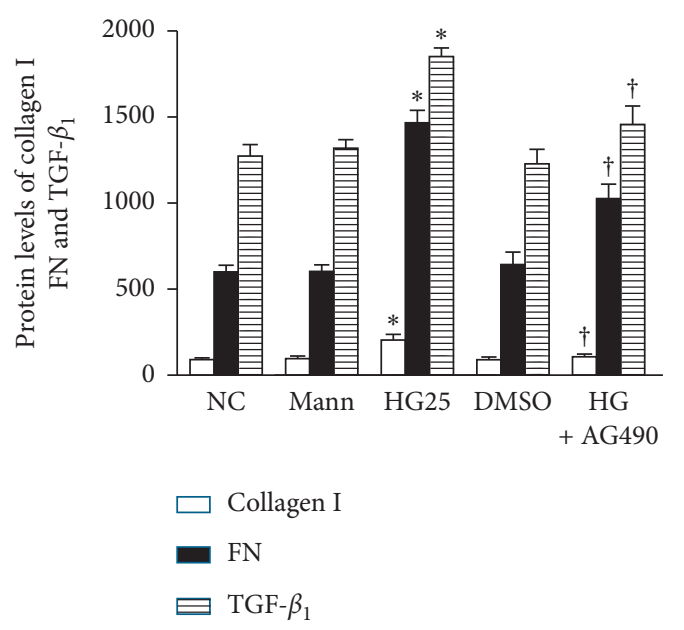

(e)

FIGURE 1: High glucose increases the protein levels of TGF- $\beta 1$, FN, and collagen I through the phosphorylation of the JAK2/STAT3 pathway $(n=3)$. (a, b) High glucose facilitated the phosphorylation of JAK2 and STAT3. (c, d) AG490 (100 $\mu$ M) inhibited JAK2/STAT3 phosphorylation and (e) reduced the overexpression of TGF- $\beta 1, \mathrm{FN}$, and collagen I proteins $\left({ }^{*} P<0.05\right.$ compared with the NC group; ${ }^{\dagger} P<0.05$ compared with the HG25 group).

coincubated with $25 \mathrm{mmol} / \mathrm{L}$ glucose for $24 \mathrm{~h}$. The cell viability was not affected by GSK2606414 (Figure 6(b)).

\section{Discussion}

$\mathrm{DKD}$ is a serious complication of diabetes, with a major morphologic feature being the change in ECM. Owing to increased amounts of ECM, basement membranes are thickened, the glomerular mesangial matrix and tubulointerstitial space are expanded, which ultimately led to failure of kidney function [15]. TGF- $\beta 1$ is the central cytokine in many cellular processes, and accumulating evidence reveals that TGF- $\beta 1$ is closely related to renal ECM deposits in DKD [16]. High glucose can upregulate TGF- $\beta 1$ 


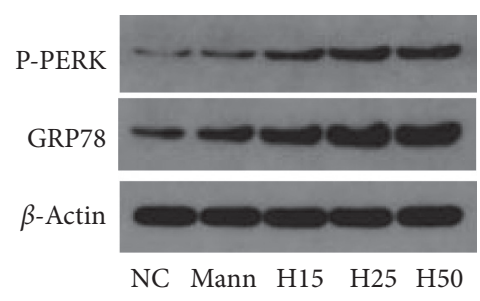

(a)

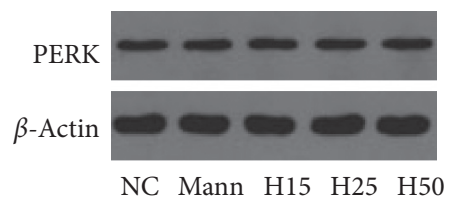

(b)

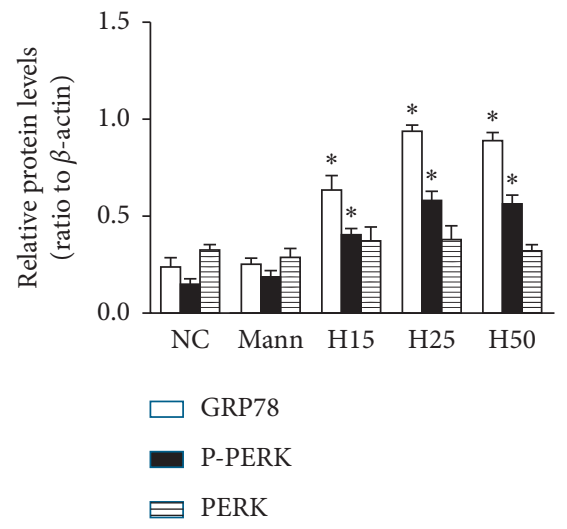

(c)

FIGURE 2: ERS is triggered by high glucose $(n=3)$. (a, c) The expression of GRP78 and phosphorylation of PERK were increased under high glucose conditions, $(\mathrm{b}, \mathrm{c})$ while PERK protein did not change $\left({ }^{*} P<0.05\right.$ compared with the NC group).

TABLE 2: The protein levels of TGF- $\beta_{1}$, FN, and collagen I in different concentrations of thapsigargin at different times.

\begin{tabular}{lccccc}
\hline & TGF- $\beta 1(\mathrm{ng} / \mathrm{L})$ & & FN $(\mu \mathrm{g} / \mathrm{ml})$ & & Coll I (ng/L) \\
& Mean \pm SD & $P$ & Mean \pm SD & $P$ & Mean \pm SD \\
\hline NC & $1214.17 \pm 107.99$ & - & $595.82 \pm 96.72$ & - & $87.53 \pm 9.22$ \\
DMSO & $1207.90 \pm 77.80$ & 1.00 & $607.90 \pm 88.25$ & 1.00 & $89.16 \pm 12.42$ \\
Thap, 0.1/6 h & $1324.93 \pm 117.29$ & 0.98 & $689.67 \pm 98.39$ & 0.95 & $117.98 \pm 26.66$ \\
Thap, 0.1/24h & $1868.72 \pm 121.23^{*}$ & $<0.05$ & $983.82 \pm 100.21^{*}$ & $<0.05$ & $190.99 \pm 25.37^{*}$ \\
Thap, 0.1/48 h & $1546.91 \pm 150.47$ & 0.20 & $755.08 \pm 109.47$ & 0.59 & $135.02 \pm 24.07$ \\
Thap, 0.2/6 h & $1318.07 \pm 181.84$ & 0.99 & $772.14 \pm 113.70$ & 0.47 & $138.21 \pm 23.80$ \\
Thap, 0.2/24h & $1794.28 \pm 157.11^{*}$ & $<0.05$ & $896.43 \pm 105.48^{*}$ & 0.04 & $157.87 \pm 20.64^{*}$ \\
Thap, 0.2/48 h & $1423.61 \pm 242.25$ & 0.70 & $744.88 \pm 119.11$ & 0.66 & 0.51 \\
\hline
\end{tabular}

$N=3,{ }^{*} P<0.05$ compared with the NC group.

protein in renal tubular epithelium, which then induces ECM accumulation and delays its degradation. The results of our experiment were consistent with those of previous studies, as TGF- $\beta 1, \mathrm{FN}$, and collagen I were all overproduced under high glucose conditions.

It is, however, unclear that how high glucose induces the expression of TGF- $\beta 1$ and ECM accumulation. The JAK/ STAT pathway is a crucial signal transduction cascade that regulates cellular activation, proliferation, and inflammation, and the activation of the JAK/STAT-signaling pathway leads to the development of fibrosis in the lung and kidney via the induction of TGF- $\beta 1$ and collagens $[17,18]$. A STAT3 inhibitor suppressed TGF- $\beta 1$ so as to prevent the occurrence of DKD in STZ-induced rats [19], while it was also reported that JAK2/STAT3 signaling may play a role in the renal fibrosis repair process in mice with UUO (unilateral ureteral obstruction) and that this effect was partially mediated by MMP-2 activation. This article suggested that TGF- $\beta 1$ may not be a molecular target of the JAK/STAT axis [20]. Therefore, to clarify the role of JAK2/STAT3 in the synthesis of TGF- $\beta 1$ and ECM, NRK-52E cells were herein cultured with high glucose and concomitantly incubated with AG490. Our results revealed that AG490 inhibited the phosphorylation of JAK2/STAT3, accompanied by a decrease in TGF- $\beta 1$ protein and ECM components. These data indicated that although the overproduction of TGF- $\beta 1$ and ECM induced by high glucose was JAK2/STAT3-dependent, the underlying mechanism is arcane and requires further elucidation.

ERS has emerged as an important pathophysiologic phenomenon that underlies metabolic diseases, such as diabetes, and GRP78 is a vital molecular indicator of ERS. The most immediate response to ER stress is the homodimerization and transphosphorylation of PERK [21]. Our previous results showed that high glucose elevated the GRP78 protein and PERK phosphorylation. As mannitol 


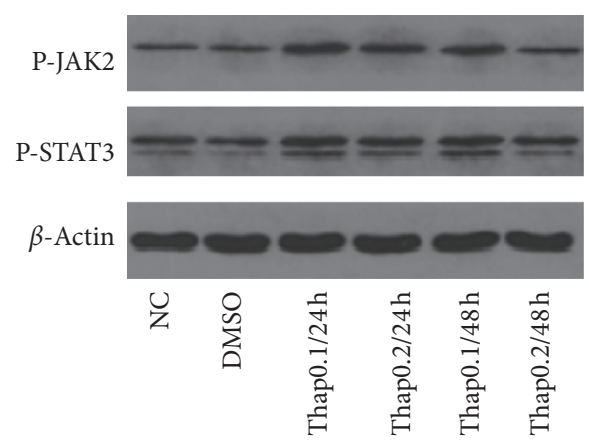

(a)

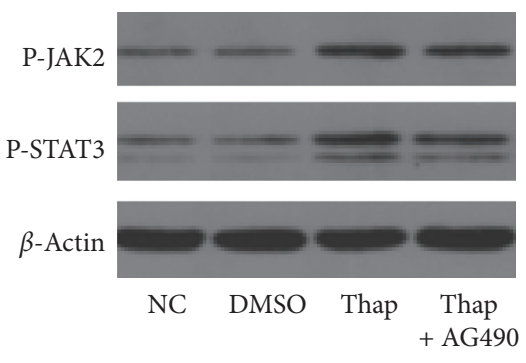

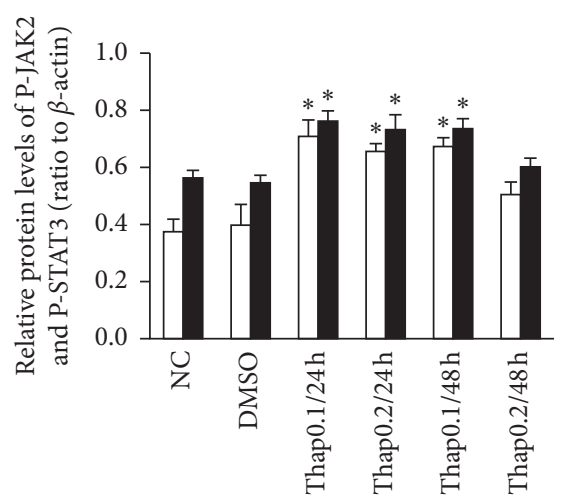

$\square$ P-JAK2

- P-STAT3

(b)

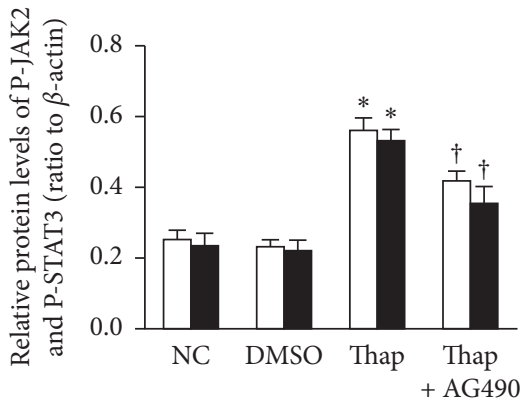

P-JAK2

P-STAT3

(d)

(c)

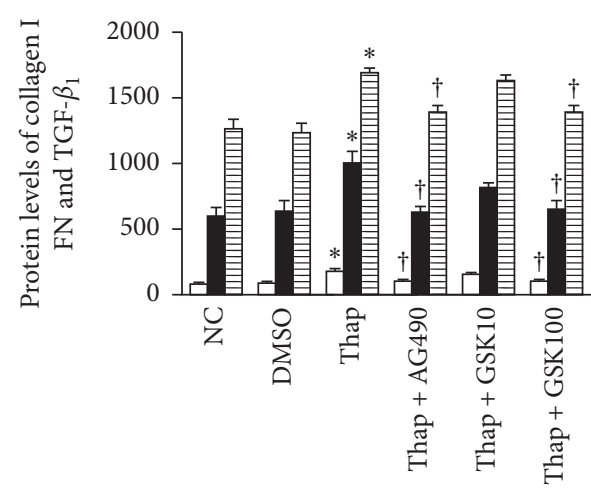

$\square$ Collagen I

FN

$\equiv$ TGF- $\beta_{1}$

(e)

Figure 3: Overexpression of TGF- $\beta 1$ and ECM deposits during ERS are JAK2/STAT3-dependent $(n=3)$. (a, b) Thapsigargin accelerated the phosphorylation of JAK2 and STAT3. (c, d) AG490 (100 $\mu \mathrm{M})$ blocked the JAK2/STAT3 phosphorylation as well as (e) the overexpression of TGF- $\beta 1$, FN, and collagen I $\left({ }^{*} P<0.05\right.$ compared with the NC group; ${ }^{\dagger} P<0.05$ compared with the thap group).

exerted no effect on either of these proteins, this suggested us that these effects were not induced by hyperosmosis [14].

Moderate ER stress can maintain cellular homeostasis. However, severe or prolonged ERS ultimately induces deterioration of cellular function and cell death. ERS has been documented as being intimately associated with inflammation and stress signaling networks [22]. There is evidence that the interactions between ERS and STAT singling may be exquisitely intertwined. ERS can trigger STAT3 in the acutephase response of infection, which may then facilitate the 


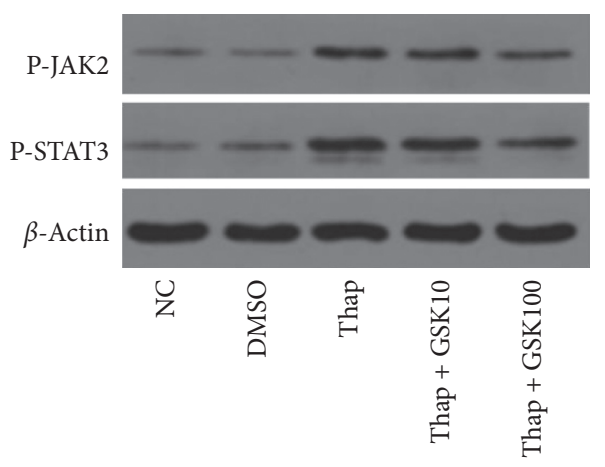

(a)

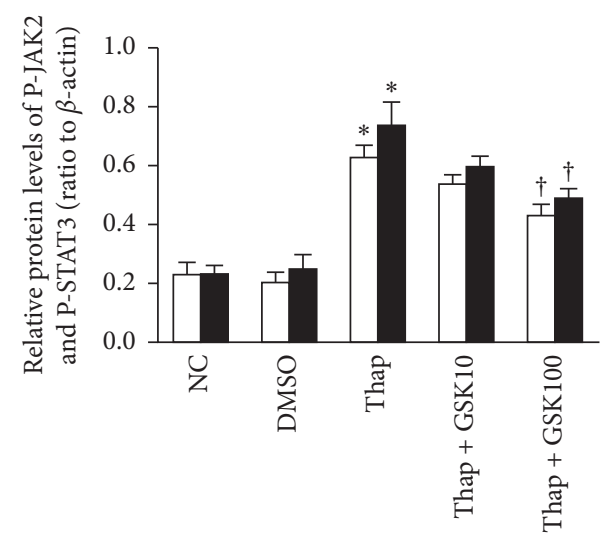

$\square$ P-JAK2

P-STAT3

(b)

FIgURE 4: The activation of JAK2/STAT3 during ERS is partially PERK-dependent $(n=3)$. Cells were pretreated with GSK2606414 for 30 minutes and cocultured with thapsigargin. GSK2606414 $(100 \mathrm{nM})$ reduced the phosphorylation of JAK2/STAT3 $\left({ }^{*} P<0.05\right.$ compared with the NC group, ${ }^{\dagger} P<0.05$ compared with the thap group).

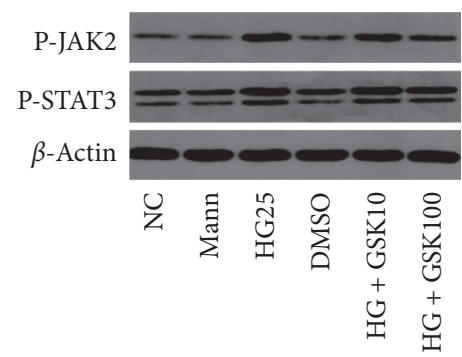

(a)

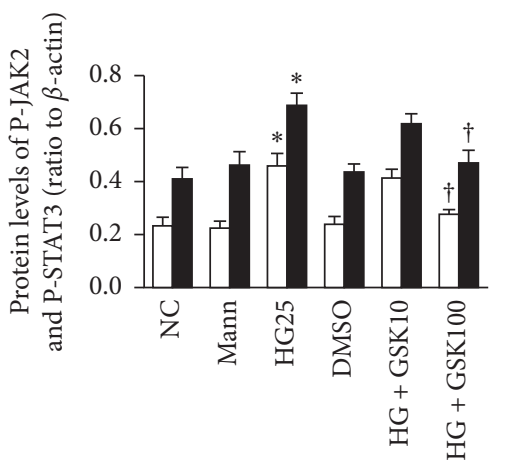

$\square$ P-JAK2

P-STAT3

(b)

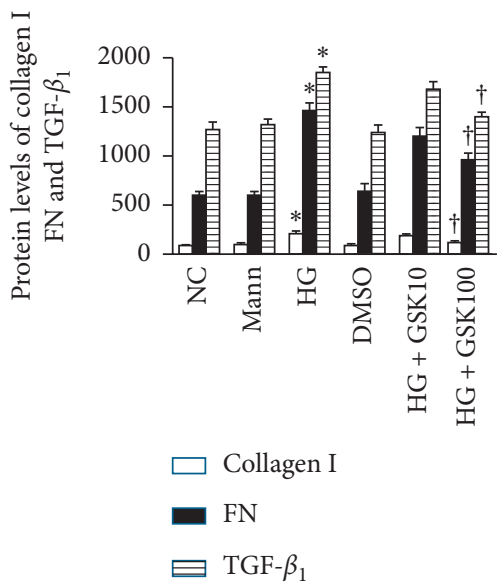

(c)

FIgURE 5: High glucose facilitates the deposit of ECM via the PERK-dependent JAK2/STAT3 pathway in NRK-52E cells ( $n=3$ ). Cells were pretreated with different concentrations of GSK2606414 (10 nM, $100 \mathrm{nM})$ for 30 minutes and coincubated with $25 \mathrm{mmol} / \mathrm{L}$ glucose for $24 \mathrm{~h}$. $(\mathrm{a}, \mathrm{b})$ The higher concentration of GSK2606414 (100 nM) inhibited the phosphorylation of JAK2 and STAT3 induced by high glucose as well as TGF- $\beta 1$, FN, and collagen I proteins (c) $\left({ }^{*} P<0.05\right.$ compared with the NC group; ${ }^{\dagger} P<0.05$ compared with the HG25 group).

processing and delivery of newly synthesized loads of acutephase proteins, and thus prevent liver from injury during infection [23]. ERS is known to activate STAT3 and NF- $\kappa \mathrm{B}$ [24]. Both PERK and IRE1 $\alpha$ can activate STAT3, which in turn promotes survival through upregulation of antiapoptotic protein [25]. PERK is specifically activated in response to ER stress in neighboring cells of intestinal stem cells (ISC) through JAK/STAT signaling, which can limit Drosophila lifespan by promoting ISC proliferation [26]. Since our study showed that thapsigargin promoted the phosphorylation of JAK2 and STAT3, we subsequently used a potent selective PERK inhibitor (GSK2606414) to pretreat cells. After coculture with GSK2606414, the increased levels of JAK2 and STAT3 induced by thapsigargin declined significantly. These results showed that ERS elevated the phosphorylation of JAK2 and STAT3 partly through the PERK branch of UPR. Additionally, TGF- $\beta 1$, FN, and collagen I proteins induced by thapsigargin were all blocked by GSK2606414. These results revealed that ERS upregulated TGF- $\beta 1$ and ECM proteins, and that these actions were partially mediated through the PERK/JAK2/STAT3 pathway.

However, it is still unclear as to how high levels of glucose induced the accumulation of ECM through ER stress in DKD. Recent studies have revealed that ERS plays a crucial role in renal fibrosis, which can be improved by amelioration of ERS. [27]. It has also been reported that ER stress appears to occupy a critical role in activation of the albuminuria-induced inflammasome. Elimination of ERS 


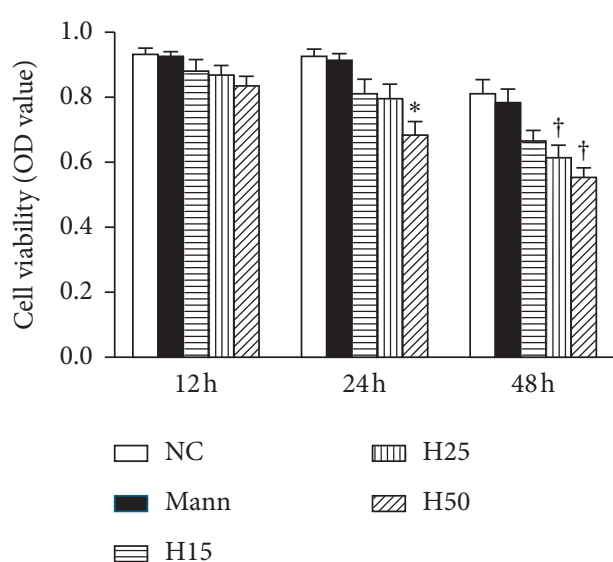

(a)

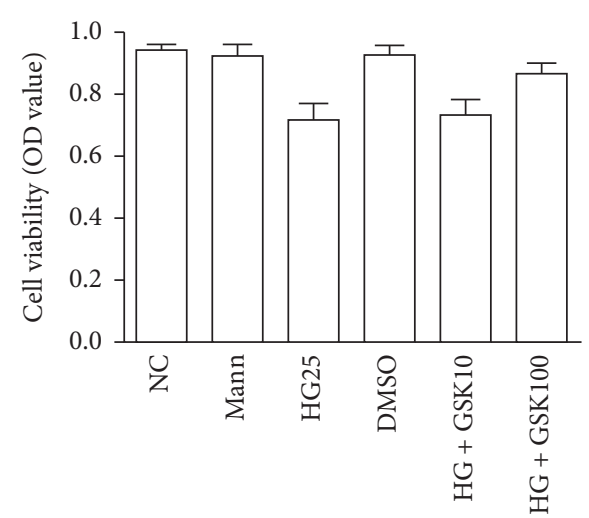

(b)

FIgURE 6: Cell viability in different concentration of glucose $(n=3)$. (a) NRK-52E cells were cultured in the medium with different concentrations of glucose or mannitol for $12 \mathrm{~h}, 24 \mathrm{~h}$, or $48 \mathrm{~h}\left({ }^{*} \mathrm{P}<0.05\right.$ compared with $5.6 \mathrm{mmol} / \mathrm{L}$ group at $24 \mathrm{~h}$; ${ }^{\dagger} P<0.05$ compared with $5.6 \mathrm{mmol} / \mathrm{L}$ group at $48 \mathrm{~h}$ ). (b) NRK-52E cells were pretreated with GSK2606414 (10 nM and $100 \mathrm{nM}$ ) for 30 minutes and coincubated with $25 \mathrm{mmol} / \mathrm{L}$ glucose for $24 \mathrm{~h}$. GSK2606414 treatment did not decrease the cell viability.

might thereby serve as a novel avenue for ameliorating kidney epithelial cell injury induced by albuminuria [28]. Qi et al. [29] demonstrated that 4-PBA exerted a notable renoprotective effect by lowering the expression of p-PERK in STZ-induced diabetic rats. Moreover, $\mathrm{P} 58^{\mathrm{IPK}}$ is a vital component whose function was ascertained by suppressing PERK activation during ERS. Yang et al. [30] also established a protective role for $\mathrm{P} 58^{\mathrm{IPK}}$ against ER stress-mediated diabetic retinopathy. In our study, when NRK-52E cells were cultured for $24 \mathrm{~h}$ with high glucose in the presence or absence of GSK2606414, we showed that GSK2606414 treatment suppressed the increase in phosphorylation of JAK2 and STAT3 induced by high glucose. We also showed that this was accompanied by the downregulation of TGF- $\beta 1$ protein and constituents of the ECM.

In conclusion, our findings revealed that high glucose induced an increase in the synthesis of TGF- $\beta 1$ and ECM deposits through the JAK2/STAT3-signaling pathway, and this increase was partially mediated by the PERK branch of the unfolded protein response in ERS. Since we traditionally recognize that TGF- $\beta 1$ and the accumulation of ECM contribute to the deterioration of renal function, our study suggests that inhibiting PERK during ER stress might constitute a potential novel therapy for improving DKD.

\section{Data Availability}

The data analyzed during this study are included within the article.

\section{Conflicts of Interest}

The authors declare that they have no conflicts of interest.

\section{Acknowledgments}

This work was supported by grants from the Natural Science Foundation of Hubei Province (no. 2017CFB779).

\section{References}

[1] A. C. Webster, E. V. Nagler, R. L. Morton, and P. Masson, "Chronic kidney disease," The Lancet, vol. 389, no. 10075, pp. 1238-1252, 2017.

[2] F. P. Schena and L. Gesualdo, "Pathogenetic mechanisms of diabetic nephropathy," Journal of the American Society of Nephrology: JASN, vol. 16, no. 1, pp. S30-S33, 2005.

[3] N. G. Docherty, M. Murphy, F. Martin, E. P. Brennan, and C. Godson, "Targeting cellular drivers and counter-regulators of hyperglycaemia- and transforming growth factor- $\beta 1$-associated profibrotic responses in diabetic kidney disease," Experimental Physiology, vol. 99, no. 9, pp. 1154-1162, 2014.

[4] A. Valladares-Salgado, J. Angeles-martínez, M. Rosas et al., "Association of polymorphisms within the transforming growth factor- $\beta 1$ gene with diabetic nephropathy and serum cholesterol and triglyceride concentrations," Nephrology, vol. 15, no. 6, pp. 644-648, 2010.

[5] X. Wang, S. Shaw, F. Amiri, D. C. Eaton, and M. B. Marrero, "Inhibition of the JAK/STAT signaling pathway prevents the high glucose-induced increase in TGF- and fibronectin synthesis in mesangial cells," Diabetes, vol. 51, no. 12, pp. 3505-3509, 2002.

[6] F. Matsui and K. K. Meldrum, "The role of the Janus kinase family/signal transducer and activator of transcription signaling pathway in fibrotic renal disease," Journal of Surgical Research, vol. 178, no. 1, pp. 339-345, 2012.

[7] F. Amiri, S. Shaw, X. Wang et al., "Angiotensin II activation of the JAK/STAT pathway in mesangial cells is altered by high glucose," Kidney International, vol. 61, no. 5, pp. 1605-1616, 2002.

[8] L. Zhu, S. Zhao, S. Liu, Q. Liu, F. Li, and J. Hao, "PTEN regulates renal extracellular matrix deposit via increased CTGF in diabetes mellitus," Journal of Cellular Biochemistry, vol. 117, no. 5, pp. 1187-1198, 2016.

[9] S. Ferrè, Y. Deng, S. C. Huen et al., "Renal tubular cell spliced $\mathrm{X}$-box binding protein 1 (Xbp1s) has a unique role in sepsisinduced acute kidney injury and inflammation," Kidney International, vol. 96, no. 6, pp. 1359-1373, 2019.

[10] K. Van, R. Mehta, T. Wang et al., "Cell surface expression of 78-kDa glucose-regulated protein (GRP78) mediates diabetic 
nephropathy," Journal of Biological Chemistry, vol. 294, no. 19, pp. 7755-7768, 2019.

[11] F.-F. Xu and X.-H. Liu, "Calreticulin translocation aggravates endoplasmic reticulum stress-associated apoptosis during cardiomyocyte hypoxia/reoxygenation," Chinese Medical Journal, vol. 128, no. 3, pp. 353-360, 2015.

[12] A. V. Cybulsky, "Endoplasmic reticulum stress, the unfolded protein response and autophagy in kidney diseases," Nature Reviews Nephrology, vol. 13, no. 11, pp. 681-696, 2017.

[13] G. P. Meares, Y. Liu, R. Rajbhandari et al., "PERK-dependent activation of JAK1 and STAT3 contributes to endoplasmic reticulum stress-induced inflammation," Molecular and Cellular Biology, vol. 34, no. 20, pp. 3911-3925, 2014.

[14] Y. Bao, Y. Ao, B. Yi, and J. Batubayier, "High levels of glucose induce epithelial-mesenchymal transition in renal proximal tubular cells through PERK-eIF2 $\alpha$ pathway," Chinese Medical Journal, vol. 132, no. 7, pp. 868-872, 2019.

[15] T. W. C. Tervaert, A. L. Mooyaart, K. Amann et al., "Pathologic classification of diabetic nephropathy," Journal of the American Society of Nephrology, vol. 21, no. 4, pp. 556-563, 2010.

[16] L. Jie, Q. Pengcheng, H. Qiaoyan et al., "Dencichine ameliorates kidney injury in induced type II diabetic nephropathy via the TGF- $\beta$ /Smad signalling pathway," European Journal of Pharmacology, vol. 812, pp. 196-205, 2017.

[17] S. Q. Zhao, Z. C. Shen, B. F. Gao, and P. Han, "microRNA-206 overexpression inhibits epithelial-mesenchymal transition and glomerulosclerosis in rats with chronic kidney disease by inhibiting JAK/STAT signaling pathway," Journal of Cellular Biochemistry, vol. 120, no. 9, pp. 14604-14617, 2019.

[18] Z. Dong, X. Zhao, W. Tai et al., "IL-27 attenuates the TGF- $\beta 1$ induced proliferation, differentiation and collagen synthesis in lung fibroblasts," Life Sciences, vol. 146, pp. 24-33, 2016.

[19] J. Chiu, Z. A. Khan, H. Farhangkhoee, and S. Chakrabarti, "Curcumin prevents diabetes-associated abnormalities in the kidneys by inhibiting p300 and nuclear factor- $\kappa \mathrm{B}$," Nutrition, vol. 25, no. 9, pp. 964-972, 2009.

[20] K. Koike, S. Ueda, S.-I. Yamagishi et al., "Protective role of JAK/STAT signaling against renal fibrosis in mice with unilateral ureteral obstruction," Clinical Immunology, vol. 150, no. 1, pp. 78-87, 2014.

[21] J. D. Malhotra and R. J. Kaufman, "Endoplasmic reticulum stress and oxidative stress: a vicious cycle or a double-edged sword?" Antioxidants \& Redox Signaling, vol. 9, no. 12, pp. 2277-2294, 2007.

[22] G. S. Hotamisligil, "Endoplasmic reticulum stress and the inflammatory basis of metabolic disease," Cell, vol. 140, no. 6 , pp. 900-917, 2010.

[23] A.-N. N. Ahyi, L. J. Quinton, M. R. Jones et al., "Roles of STAT3 in protein secretion pathways during the acute-phase response," Infection and Immunity, vol. 81, no. 5, pp. 1644-1653, 2013.

[24] A. D. Garg, A. Kaczmarek, O. Krysko, P. Vandenabeele, D. V. Krysko, and P. Agostinis, "ER stress-induced inflammation: does it aid or impede disease progression?" Trends in Molecular Medicine, vol. 18, no. 10, pp. 589-598, 2012.

[25] J. R. Cubillos-Ruiz, S. E. Bettigole, and L. H. Glimcher, "Tumorigenic and immunosuppressive effects of endoplasmic reticulum stress in cancer," Cell, vol. 168, no. 4, pp. 692-706, 2017.

[26] L. Wang, H. D. Ryoo, Y. Qi, and H. Jasper, "PERK limits Drosophila lifespan by promoting intestinal stem cell proliferation in response to ER stress," Plos Genetics, vol. 11, no. 5, Article ID e1005220, 2015.
[27] Q. F. Liu, J. M. Ye, Z. Y. Deng, L. X. Yu, Q. Sun, and S. S. Li, "Ameliorating effect of Klotho on endoplasmic reticulum stress and renal fibrosis induced by unilateral ureteral obstruction," Iranian Journal of Kidney Diseases, vol. 9, no. 4, pp. 291-297, 2015.

[28] L. Fang, D. Xie, X. Wu, H. Cao, W. Su, and J. Yang, "Involvement of endoplasmic reticulum stress in albuminuria induced inflammasome activation in renal proximal tubular cells," Plos One, vol. 8, no. 8, Article ID e72344, 2013.

[29] W. Qi, J. Mu, Z.-F. Luo et al., “Attenuation of diabetic nephropathy in diabetes rats induced by streptozotocin by regulating the endoplasmic reticulum stress inflammatory response," Metabolism, vol. 60, no. 5, pp. 594-603, 2011.

[30] H. Yang, R. Liu, Z. Cui et al., "Functional characterization of 58-kilodalton inhibitor of protein kinase in protecting against diabetic retinopathy via the endoplasmic reticulum stress pathway," Molecular Vision, vol. 17, pp. 78-84, 2011. 